\title{
A multiple-center phase II study of weekly docetaxel and oxaliplatin as first-line treatment in patients with advanced gastric cancer
}

\author{
Jen-Shi Chen $\cdot$ Yen-Yang Chen $\cdot$ Jen-Sheng Huang $\cdot$ Kun-Yun Yeh $\cdot$ \\ Ping-Tsung Chen • Wen-Chi Shen · Hung-Chih Hsu • Yung-Chung Lin • \\ Hung-Ming Wang
}

Received: 8 February 2011/Accepted: 2 May 2011/Published online: 8 June 2011

(C) The International Gastric Cancer Association and The Japanese Gastric Cancer Association 2011

\begin{abstract}
Background Docetaxel and oxaliplatin are active agents for advanced gastric cancer (GC). The combination of these two drugs in a triweekly schedule is an active and attractive regimen for gastric cancer but with significant hematological toxicities. A multicenter phase II study was designed to establish an active regimen with good tolerability by using a weekly docetaxel-oxaliplatin (DO) combination in GC patients.
\end{abstract}

Part of the result of this study was presented at ECCO 15/ESMO 34 on September 23, 2009, in Berlin, Germany.

J.-S. Chen · W.-C. Shen · H.-C. Hsu · Y.-C. Lin · H.-M. Wang Division of Hematology-Oncology, Department of Internal Medicine, Linkou Chang Gung Memorial Hospital,

Taoyuan, Taiwan

Y.-Y. Chen

Division of Hematology-Oncology, Department of Internal Medicine, Kaohsiung Chang Gung Memorial Hospital,

Kaohsiung, Taiwan

J.-S. Huang · K.-Y. Yeh

Division of Hematology-Oncology, Department of Internal

Medicine, Keelung Chang Gung Memorial Hospital,

Keelung, Taiwan

P.-T. Chen

Division of Hematology-Oncology, Department of Internal

Medicine, Chiayi Chang Gung Memorial Hospital,

Chiayi, Taiwan

J.-S. Chen $(\bowtie)$

Division of Hematology-Oncology, Department of Internal

Medicine, Chang Gung Memorial Hospital, Chang Gung

University College of Medicine, No 5 Fu-Hsing Road,

Kuei-Shan, Taoyuan 333, Taiwan

e-mail: js1101@adm.cgmh.org.tw
Methods Eligible patients had histologically confirmed stage IV gastric cancer without previous palliative chemotherapy; age $\geq 18$ years; Eastern Cooperative Oncology Group (ECOG) performance status $\leq 2$; at least one measurable lesion; and adequate hematological, renal, and liver functions. All patients received premedications with dexamethasone and 5-HT3 antagonist before the chemotherapy. Docetaxel (Taxotere ${ }^{\circledR}$; Sanofi-Aventis) $30 \mathrm{mg} / \mathrm{m}^{2}$ followed by oxaliplatin (Eloxatin ${ }^{\circledR} ;$ Sanofi-Aventis) $65 \mathrm{mg} / \mathrm{m}^{2}$ were administered on days 1 and 8 of each 21-day cycle. Treatment continued until disease progression, intolerable toxicity, or consent withdrawal. Toxicities were graded according to the National Cancer Institute Common Toxicity Criteria (NCI-CTC) version 3.0. Tumor responses were evaluated every 2 cycles by the Response Evaluation Criteria in Solid Tumors Guidelines.

Results From May 2007 to December 2008, a total of 47 patients were enrolled. There were 8 females and 39 males with a median age of 57 years (range 26-76). Forty-three patients were evaluable for response. Two patients obtained a complete response $(4.7 \%)$ and 12 patients had a partial response (27.9\%), with an overall response rate of $32.6 \%$ (95\% confidence interval [CI] 19.1-48.5); 20 patients experienced stable disease (46.5\%), and the disease progressed in 9 patients (20.9\%). Median time to disease progression was 4.2 months and median overall survival was 8.3 months. All 47 patients were assessable for toxicity. Major grade 3/4 hematological toxicities were anemia (5 patients, $10.6 \%$ ), neutropenia ( 2 patients, $4.3 \%$ ), and leukopenia ( 1 patient, $2.1 \%$ ). The most common grade $3 / 4$ nonhematological toxicities were fatigue (3 patients, $6.4 \%$ ) and aspartate aminotransferase (AST) elevation in 3 patients $(6.4 \%)$.

Conclusions The combination of weekly DO demonstrated a well-tolerated profile with moderate activity in the 
treatment of advanced gastric cancer. Further studies of the combination together with a fluoropyrimidine are warranted.

Keywords Chemotherapy - Docetaxel · Oxaliplatin ·

Gastric cancer

\section{Introduction}

Gastric cancer is one of the leading causes of cancer death worldwide [1]. Surgery is the only curative treatment for localized disease, but the 5-year overall survival (OS) rate remains dismal for patients with advanced or metastatic disease [2]. Systemic chemotherapy is often used in patients with advanced or metastatic disease, since it has been associated with a significantly superior median survival and a better quality of life in comparison with best supportive care alone [3]. However, the prognosis for advanced disease is still poor, with a 5-year survival of $<10 \%$, and chemotherapy in this setting remains palliative. At present, cisplatin (CDDP)-based and 5-fluorouracil (5FU)-based regimens are commonly used [4]. Although these regimens yield significant response rates (up to 50\%), the median time to progression (TTP) and median survival are consistently modest, at around 4-5 and 8-11 months, respectively. The development of new chemotherapeutic regimens to achieve a higher tumor response rate and better toxicity profiles is urgently needed [4].

Docetaxel (D), a mitotic inhibitor, is an active agent in several solid malignancies, including non-small cell lung cancer, breast cancer, and head and neck cancers [5]. It has also been shown to have promising activity in advanced gastric cancer, with a single-agent response rate of $17-24 \%$ in the front-line setting $[6,7]$. In a randomized phase III trial (V325 study) involving 457 patients, the combination of D $75 \mathrm{mg} / \mathrm{m}^{2}$ plus CDDP $75 \mathrm{mg} / \mathrm{m}^{2}$ on day 1 plus $5-\mathrm{FU}$ $750 \mathrm{mg} / \mathrm{m}^{2} /$ day by continuous intravenous infusion over 5 days every 3 weeks (DCF) was compared with CDDP $100 \mathrm{mg} / \mathrm{m}^{2}$ on day 1 followed by 5 -day infusion of 5 -FU $1000 \mathrm{mg} / \mathrm{m}^{2} /$ day every 4 weeks (CF) [8]. The median TTP was longer with DCF compared to CF (5.6 vs. 3.7 months; risk reduction $32 \%$; log-rank $p=0.0004$ ). With a median follow-up of 23 months, OS was improved with DCF (risk reduction $23 \%$, log-rank $p=0.0201$ ), with a 2-year survival of $18 \%$ with DCF versus $9 \%$ with $\mathrm{CF}$. The response rate with DCF (37\%) was superior to that with CF $(25 \%$; $p=0.0106$ ) [8]. The V325 study demonstrated that adding $\mathrm{D}$ to a frequently used regimen of CDDP and 5-FU (CF) provided benefits with regard to OS, response rate, time-todisease progression, clinical benefit, and health-related quality of life. Although the DCF regimen provides these advantages, it is accompanied by an increase in toxicity compared with the doublet regimen. More recently, weekly administration of $D$ at doses of $36-42 \mathrm{mg} / \mathrm{m}^{2} /$ week has been used increasingly because of its lesser myelosuppression than the triweekly schedule [9]. Only $10-20 \%$ of patients receiving a weekly schedule of $\mathrm{D}$ developed grade 3 or 4 neutropenia. A phase III study in non-small cell lung cancer showed that weekly D had a less toxic profile than a triweekly schedule and the efficacy was similar to that of a triweekly schedule [10]. In a phase II study of weekly D reported by Graziano et al. [11], $36 \mathrm{mg} / \mathrm{m}^{2} /$ week as second-line therapy for patients with gastric cancer showed activity with low bone marrow toxicity. Therefore, weekly D may offer a new therapeutic option in gastric cancer due to its improved therapeutic index.

Oxaliplatin (OXA) is a third-generation platinum compound that has a wide range of antitumor activities. Compared with CDDP, OXA appears to have a better safety profile, and cross-resistance to CDDP is minimal [12]. A phase III study from United Kingdom (REAL-2 trial) suggests that substituting OXA for CDDP may result in a more effective and well-tolerated combination [13]. A study by the Arbeitsgemeinschaft Internistische Onkologie (AIO) was designed to compare FLO [5-FU, leucovorin (LV), and OXA] with FLP (5-FU, LV, and CDDP) in patients with advanced gastric cancer [14]. The result showed that FLO had reduced toxicity as compared with FLP. In older adult patients, FLO also seemed to be associated with improved efficacy.

From the literature, there is a strong rationale for administering OXA and D in combination for gastric cancer. These agents have distinct and complementary mechanisms of action, potential lack of cross-resistance, and different toxicity and favorability profiles. To date, several phase II trials have been conducted to assess the potential of triweekly docetaxel-oxaliplatin (DO) combinations in advanced gastric cancer [15-17]. The US Oncology Group study $(n=71)$ showed an overall response rate of $47 \%$ with first-line $\mathrm{D} 60 \mathrm{mg} / \mathrm{m}^{2}$ on day 1 plus OXA $130 \mathrm{mg} / \mathrm{m}^{2}$ on day 1 every 3 weeks until progression, but with a significant incidence of hematological toxicity (68\% grade 3/4 neutropenia and $6 \%$ febrile neutropenia) [15]. DO represents a promising new combination for gastric cancer chemotherapy and we want to establish an optimal treatment regimen based on this combination. The logical next step is to establish a more tolerable dosing schedule based on this combination. With these profiles described above, it appears that a combination of weekly DO may be an effective and well-tolerated regimen for advanced gastric cancer. Currently, no literature has provided experiences in this combination setting.

The dosing selected for this trial was based on the dose equivalence in the US Oncology Group Study [15]. The dose-intensity (DI) of D $30 \mathrm{mg} / \mathrm{m}^{2}$ on days 1 and 8 every 3 weeks is as the same as that of $60 \mathrm{mg} / \mathrm{m}^{2}$ day 1 every 3 
weeks, while the dose-intensity of OXA $65 \mathrm{mg} / \mathrm{m}^{2}$ on days 1 and 8 every 3 weeks is as the same as that of $130 \mathrm{mg} / \mathrm{m}^{2}$ day 1 every 3 weeks. This forms the rationale of the current phase II study. Herein, we report our study results.

\section{Patients and methods}

\section{Eligibility criteria}

Eligibility criteria were as follows: patients had to have a histological or cytological diagnosis of gastric adenocarcinoma with metastatic disease. Patients had to be $\geq 18$ and $\leq 75$ years of age and have an Eastern Cooperative Oncology Group (ECOG) performance status score of $\leq 2$. Patients had to have recovered from recent surgery (procedure at least 2 weeks prior to enrollment) or radiotherapy (completed at least 4 weeks prior to enrollment) and to have not had any palliative chemotherapy. Patients with previous adjuvant and/or neoadjuvant chemotherapy were eligible if they had not been treated for more than 6 months between the end of the adjuvant chemotherapy and the first relapse. Patients had to have at least one measurable lesion at enrollment. Measurable lesions were defined as lesions that could be measured in at least one dimension as $\geq 20 \mathrm{~mm}$ with a conventional technique or as $\geq 10 \mathrm{~mm}$ with spiral computed tomography (CT). Previously irradiated lesions were not considered measurable target lesions. Patients were allowed to complete their initial work-up within 2 weeks prior to receiving the first cycle of chemotherapy in this trial.

Patients had to show baseline eligibility in laboratory tests as follows: neutrophils $\geq 1500 / \mathrm{L}$, platelets $\geq 100,000 /$ L, serum creatinine $\leq 1.5 \times$ upper limit of normal (ULN), total bilirubin $\leq 1.5 \times$ ULN, SGOT and SGPT $\leq 2.5 \times$ ULN $(\leq 5.0 \times \mathrm{ULN}$ if hepatic metastasis was present). Finally, patients had to complete an informed consent form before entering the study. This study enrolled patients at four branches of the Chang Gung Memorial Hospital in both northern and southern Taiwan. This study was approved prior to implementation by the Scientific and Research Ethics Committees of the participating institutions.

\section{Exclusion criteria}

Patients with the following conditions were excluded: life expectancy $<3$ months, had central nervous system metastasis, had bone metastasis only, pregnant or breastfeeding, clinically detectable peripheral neuropathy more than grade 2 due to any causes, concomitant illness that might be exacerbated by chemotherapy, active cardiac disease within 6 months preceding entry to the study (e.g., angina or myocardial disease), history of other malignancy except for curatively treated non-melanoma skin cancer or cervical carcinoma in situ, mental status unfit for clinical trials, or hypersensitive to any components of this chemotherapeutic regimen.

Treatment schedule

Premedication with dexamethasone $10 \mathrm{mg}$ intravenous and a serotonin receptor antagonist (5-HT3) was given before chemotherapy. Administration consisted of docetaxel (Taxotere $^{\circledR}$; Sanofi-Aventis) $30 \mathrm{mg} / \mathrm{m}^{2}$ in normal saline $250 \mathrm{ml}$ as a 1-h infusion and oxaliplatin (Eloxatin ${ }^{\circledR}$; Sanofi-Aventis) $65 \mathrm{mg} / \mathrm{m}^{2}$ in $5 \%$ glucose $250 \mathrm{ml}$ as a 2 -h infusion on day 1 and day 8 every 21 days. This regimen was repeated every 21 days as one treatment course. Dose level modification is shown in Table 1. Treatment was continued until objective evidence of disease progression was obtained, protocol noncompliance was established, or an individual patient wished to withdraw from the study at his/her own request or if an investigator felt that patient withdrawal was in the patient's best interest (for example, if surgery became acceptable).

Study evaluation

Tumor response was evaluated after every two courses of chemotherapy according to the Response Evaluation Criteria in Solid Tumors (RECIST) Guidelines [18]. Adverse events were to be recorded throughout the study, and were graded based on the National Cancer Institute Common Toxicity Criteria (NCI-CTC, version 3, 2003).

\section{Statistical considerations}

The primary endpoint was the response rate. Secondary endpoints were TTP, OS time, and drug-related toxicity. TTP

Table 1 Dose modification

\begin{tabular}{lll}
\hline & Docetaxel $\left(\mathrm{mg} / \mathrm{m}^{2}\right)$ & Oxaliplatin $\left(\mathrm{mg} / \mathrm{m}^{2}\right)$ \\
\hline Initial dose & 30 & 65 \\
Dose reduction 1 & 25 & 60 \\
Dose reduction 2 & 20 & 55
\end{tabular}

In the case of febrile neutropenia or grade 3-4 neutropenic infection or grade 4 neutropenia, both docetaxel and oxaliplatin doses will be reduced by one level. If grade 4 thrombocytopenia or grade 3 thrombocytopenia with concomitant bleeding occurs, docetaxel and oxaliplatin should be reduced by one dose level. If any grade 3 nonhematological toxicity occurs (except for nausea/vomiting and alopecia), the doses of docetaxel and oxaliplatin should be reduced one level down for all subsequent cycles. All patients were only allowed 2 dose reductions

Dose delays are based upon counts on the scheduled day of treatment. A cycle will be considered as "delayed" if it cannot be started on day $21 \pm 3$ days. If there is no recovery within 2 weeks' delay, the patient will be considered off the study treatment 
was defined as the period from the first day of drug treatment to the date when progressive disease or relapse was clearly documented. OS represented the number of days until death from any cause. Safety variables included toxicity grading, adverse events, and laboratory values. Simon's optimal twostage design was used to allow early termination if sufficient evidence of non-effectiveness was collected. The combination regimen of docetaxel plus oxaliplatin (DO) will be considered ineffective if the proportion of response is $20 \%$ or lower, and will be considered worthy of further study if the proportion of response is $40 \%$ or higher. Controlling the risk of accepting this treatment regimen when it is ineffective to be at most $20 \%, 13$ patients will be enrolled in the first stage. If three or fewer objective responses are observed, the study will be terminated. Otherwise, an additional 30 patients will be accrued in the second stage. The treatment regimen will be rejected if 12 or fewer objective responses are observed in at least 43 patients. With an estimated dropout rate of 10\%, 14 patients and 33 patients will be enrolled in the first stage and second stage, respectively.

\section{Results}

\section{Patient characteristics}

A total of 47 patients were enrolled from May 2007 to December 2008 at three sites. Of the 47 eligible patients,

Table 2 Patient characteristics, $n=47$

\begin{tabular}{ll}
\hline Characteristics & Number of patients \\
\hline Gender: female/male & $8(17 \%) / 39(83 \%)$ \\
Age, years: median (range) & $57(26-76)$ \\
ECOG PS & $18(38.3 \%)$ \\
0 & $27(57.4 \%)$ \\
1 & $2(4.3 \%)$ \\
2 & \\
Prior surgery & $12(25.5 \%)$ \\
Yes & $35(74.5 \%)$ \\
No & 47 \\
Eligible & 43 \\
Evaluable & 4 \\
Non-evaluable & \\
Causes (before 1st evaluation) & 1 \\
Patient withdrawal & 1 \\
Acute appendicitis & 1 \\
Pneumonia (death) & 1 \\
Suspected CNS infection & $220(4,1-10)$ \\
Number of treatment cycles (median, range)
\end{tabular}

$\overline{E C O G}$ PS Eastern Cooperative Oncology Group performance status, CNS central nervous system there were 8 women and 39 men (median age 57 years; range 26-76). Patient characteristics are shown in Table 2. There were 220 treatment cycles in total, with the median number of cycles being 4 (range 1-10). There were 40 instances of treatment delays in 17 patients during the 220 cycles. The relative DIs of OXA and D were $40.97 \mathrm{mg} / \mathrm{m}^{2} /$ week $(94.6 \%)$ and $18.8 \mathrm{mg}(94.7 \%)$, respectively. The dose level was reduced for OXA in 4 patients on 5 occasions and it was reduced for $\mathrm{D}$ in 5 patients on 6 occasions during the treatments.

\section{Efficacy}

By the end of December 2009, all participants had finished receiving the study treatment. Four patients were not evaluated for response before the first response evaluation: one died of cancer-related gastrointestinal (GI) bleeding; one patient died of chronic bronchitis with secondary pneumonia; one patient suffered from acute appendicitis; and one patient withdrew from the study. Forty-three patients were evaluable for response. Two patients obtained a complete response $(4.7 \%)$ and 12 patients had a partial response $(27.9 \%)$, with an overall response rate of $32.6 \%(95 \%$ confidence interval [CI] 19.1-48.5\%); 20 patients experienced stable disease $(46.5 \%)$, and the disease progressed in 9 patients $(20.9 \%)$. Median time to disease progression was 4.2 months and median OS was 8.3 months. The 1-year survival rate was $32 \%$. The most common causes for discontinuation from the study treatment were disease progression in 21 patients $(44.7 \%)$, adverse events in 16 patients $(34 \%)$, and investigator direction in 6 patients (12.8\%); 4 patients (8.5\%) were lost to follow-up (Table 3).

\section{Adverse events}

All 47 patients were assessable for adverse events (Table 4). There were 2 early deaths after the first treatment. The most common hematological toxicity was anemia and the most common non-hematological toxicity was fatigue. The most common grade $3 / 4$ toxicity was anemia $(5 / 47,10.6 \%)$, followed by fatigue $(3 / 47,6.4 \%)$, liver $(3 / 47,6.4 \%)$, neutropenia $(2 / 47,4.3 \%)$, and numbness $(2 / 47,4.3 \%)$.

\section{Discussion}

This study addresses the activity and safety of the combination of weekly D with OXA in patients with untreated metastatic gastric cancer. Our experience confirms that a combination of weekly D with OXA is feasible and shows activity. The incidence of major grade 3/4 hematological and non-hematological events was $<10 \%$. Weekly D and OXA indeed demonstrated relatively low toxicity, and the 
most common adverse event was fatigue in $70 \%$ patients, but in most of them it was grade I. Several phase II studies have been reported for D and OXA triweekly schedules for first- and second-line treatment in patients with advanced

Table 3 Patient treatment results

\begin{tabular}{|c|c|}
\hline Results & Number of patients \\
\hline \multicolumn{2}{|l|}{ Best response rate } \\
\hline Complete response (CR) & $2(4.7 \%)$ \\
\hline Partial response $(\mathrm{PR})$ & $12(27.9 \%)$ \\
\hline Stable disease (SD) & $20(46.5 \%)$ \\
\hline Progressive disease & $9(20.9 \%)$ \\
\hline Non-evaluable & 4 \\
\hline Overall response $(\%)$ & $14(32.6 \%)(95 \%$ CI 19.1-48.5) \\
\hline $\mathrm{CR}+\mathrm{PR}$ & \\
\hline Disease control rate $(\%)$ & $34(79.1 \%)(95 \%$ CI 64-90) \\
\hline $\mathrm{CR}+\mathrm{PR}+\mathrm{SD}$ & \\
\hline $\begin{array}{l}\text { Median time to disease } \\
\text { progression }\end{array}$ & $\begin{array}{l}126 \text { days ( } 4.2 \text { months) }(95 \% \text { CI } \\
109-171)\end{array}$ \\
\hline Median overall survival & $\begin{array}{l}249 \text { days ( } 8.3 \text { months) (95\% CI } \\
183-313)\end{array}$ \\
\hline 1-Year survival rate & $32 \%$ \\
\hline \multicolumn{2}{|l|}{ Off-therapy causes } \\
\hline $\begin{array}{l}\text { Objective disease } \\
\text { progression }\end{array}$ & 21 \\
\hline Lost to follow-up & 4 \\
\hline Intolerable adverse events & 16 \\
\hline Investigator's discretion & 6 \\
\hline
\end{tabular}

CI confidence interval gastric cancer [15-17, 19, 20]. Richards et al. reported their response rate was $36 \%$ and the stable disease (SD) rate was $49 \%$. The median progression-free survival (PFS) was 4.3 months and OS was 8.5 months. However, grade 3-4 neutropenia occurred in $70 \%$ of patients, although only five patients $(7 \%)$ developed febrile neutropenia [15]. In a similar phase II trial of a triweekly DO schedule from Korea [16], the results showed an overall response rate of $45.2 \%$, and the median TTP and median OS were 5.7 (95\% CI; 4.3-7.2) months and 9.9 (95\% CI; 7.8-12.0) months, respectively. In this Korean study, grade $3 / 4$ neutropenia occurred in 11 patients $(26.1 \%)$ and febrile neutropenia was observed in four patients $(9.5 \%)$. The most common non-hematological toxicity was fatigue (grade 1/2, 61.9\%). In a phase I/II study from Korea with D and OXA, OXA $130 \mathrm{mg} / \mathrm{m}^{2}$ and $\mathrm{D} 75 \mathrm{mg} / \mathrm{m}^{2}$ were recommended for the phase II study [19]. In regard to efficacy, the overall response rate was $55.3 \%$ (95\% CI, 40.6-70.1\%). The median OS was 12.7 months (95\% CI 10.4-14.9). The most common hematological toxicity was grade 3-4 neutropenia, which occurred in 11 patients (23.4\%) and in 13 of 259 cycles (5.0\%); febrile neutropenia was observed in 7 patients (14.9\%). Triweekly D and OXA as second-line treatment for advanced gastric cancer has also shown activity, but grade $3 / 4$ bone marrow toxicity was more than $20 \%[17,20]$. Based on our findings and the literature, a D and OXA combination in weekly or triweekly schedules in patients with gastric cancer is feasible and active, but there is less bone marrow toxicity in a weekly schedule.

In the present study, the major grade $3 / 4$ hematological toxicity was $<10 \%$ and this was similar to data in the report

Table 4 Major adverse events in the study patients (NCI-CTC version 3), $n=47$

\begin{tabular}{lcllll}
\hline & Grade 1 & Grade 2 & Grade 3 & Grade 4 & $\%$ of Grade 3 and Grade 4 \\
\hline Anemia & $25(53.2 \%)$ & $12(25.5 \%)$ & $5(10.6 \%)$ & 0 & 10.6 \\
WBC & $10(21.2 \%)$ & $1(2.1 \%)$ & $1(2.1 \%)$ & 0 & 2.1 \\
Neutrophils & $4(8.5 \%)$ & $7(14.9 \%)$ & 0 & $2(4.3 \%)$ & 4.3 \\
Platelets & $10(21.3 \%)$ & 0 & 0 & 0 & 0 \\
Anorexia & $17(36.2 \%)$ & 0 & $1(2.1 \%)$ & 0 & 2.1 \\
Nausea & $18(38.3 \%)$ & 0 & 0 & 0 & 0 \\
Vomiting & $11(23.4 \%)$ & $3(6.4 \%)$ & $1(2.1 \%)$ & 0 & 2.1 \\
Diarrhea & $9(19.1 \%)$ & $4(8.5 \%)$ & $1(2.1 \%)$ & 0 & 2.1 \\
Mucositis & 0 & $1(2.1 \%)$ & 0 & 0 & 0 \\
Hand-foot syndrome & $6(12.8 \%)$ & $1(2.1 \%)$ & 0 & 0 & 0 \\
Fatigue & $29(61.7 \%)$ & $2(4.3 \%)$ & $3(6.4 \%)$ & 0 & 6.4 \\
Edema & $6(12.8 \%)$ & 0 & $1(2.1 \%)$ & 0 & 2.1 \\
Numbness & $18(38.3 \%)$ & $1(2.1 \%)$ & $2(4.3 \%)$ & 0 & 4.3 \\
Alopecia & $7(14.9 \%)$ & 0 & - & - & - \\
Constipation & $7(14.9 \%)$ & $2(4.3 \%)$ & 0 & 0 & 0 \\
Liver & $26(55.3 \%)$ & $3(6.4 \%)$ & $2(4.3 \%)$ & 0 & 0 \\
Renal & $4(8.5 \%)$ & 0 & $0.1 \%)$ & 0.4 \\
\hline
\end{tabular}


of Graziano et al. [11]. However, fatigue was a common adverse event in this weekly schedule. In the literature, fatigue is indeed reported as a common adverse event and might be a barrier to the practical application of a weekly D schedule. This suggests that there is room for optimizing the schedule of D and OXA for planning subsequent treatments in gastric cancer [7]. The results of the V325 trial demonstrated that the addition of $\mathrm{D}$ to $\mathrm{CF}$ resulted not only in a higher response rate, but also in prolonged TTP and OS, improved quality of life, and greater clinical benefit [8]. However, the high incidence of grade 3-4 neutropenia (82\%) and febrile neutropenia (29\%) observed in the V325 study remains a concern. Modification of the DCF regimen to improve the toxicity profile while maintaining activity is an important issue [21]. Weekly infusion of $\mathrm{D}$ has become a good alternative to a triweekly D schedule.

A randomized phase II study of triweekly D with CDDP in gastric cancer has been reported [22]. The major grade $3 / 4$ toxicity was that about $76 \%$ of the 38 patients had neutropenia, and $21 \%$ had neutropenic fever. The efficacy was also lower than that of D-CDDP and 5-FU. Triweekly $\mathrm{D}$ with CDDP was not recommended for further study. The REAL and AIO studies [12,13] have shown that OXA has activity similar to that of CDDP in advanced gastric cancer, with less toxicity. It seems reasonable to combine $\mathrm{D}$ with OXA (replacing CDDP) in further studies.

In the present study, weekly D and OXA showed much lower hematological and non-hematological toxicity than triweekly D and OXA, but the efficacy of this regimen seems inferior to regimens that contain fluoropyrimidine. Based on these findings, a regimen of further weekly D and OXA with a fluoropyrimidine is worthy of further exploration. Weekly D $30 \mathrm{mg} / \mathrm{m}^{2}$ on days 1 and 8 in combination with an oral fluoropyrimidine (capecitabine) $825 \mathrm{mg} /$ $\mathrm{m}^{2}$ twice a day on days $1-14$ every 21 days demonstrated an overall response rate of $32 \%$, and median survival was 10 months [23]. The major grade $3 / 4$ adverse events were diarrhea in 5 patients $(13 \%)$ and hand-foot syndrome in 5 patients $(13 \%)$. At present, OXA with oral capecitabine has become one of the common options in the first-line treatment of metastatic gastric cancer [13, 24, 25]. Weekly D combined with OXA and an oral 5-fluoropyrimidine may have good potential for further study to modify the high toxicity of DCF. A Taiwan multiple center phase II study of weekly D $30 \mathrm{mg} / \mathrm{m}^{2}$ combined with CDDP $30 \mathrm{mg} / \mathrm{m}^{2}$ and oral tegafur-uracil/leucovorin on days 1-14 every 21 days has been reported recently [26]. In this study the overall response rates of the evaluable and intent-to-treat (ITT) populations were 58\% (95\% CI 41-74) and 53\% (95\% CI 38-68), respectively. In the ITT analysis, the median TTP and OS were 6.8 and 13.9 months, respectively. However, the major grade 3-4 toxicities were neutropenia $(51 \%)$, anemia $(22 \%)$, diarrhea $(16 \%)$, and infections $(20 \%)$. No patient died of treatment-related toxicities. Weekly D and platinum with an oral fluoropyrimidine may be an alternative step for DCF modification. Recently, a phase III (ToGA) study demonstrated that trastuzumab in combination with chemotherapy could be considered as a new standard option for patients with HER2-positive advanced gastric or gastro-esophageal junction cancer [27]. In the ToGA study, the response rate of the chemotherapy arm with CDDP and capecitabine was only $35 \%$ and with $30 \%$ grade $3 / 4$ neutropenia, that result was not so impressive. The search for a good partner with the targeted agent is still an important issue at the present time, and chemotherapy with better efficacy and lower toxicity will be a good option to improve the outcome of advanced gastric cancer in the future.

In conclusion, a weekly D and OXA combination for advanced gastric cancer is a good new option, and further studies of the combination together with a fluoropyrimidine are warranted.

Acknowledgments I would like to show my appreciation toward the patients who participated in this study and their families. I also would like to show my great gratitude to all dedicated investigators and research nurses. Finally, I would like to thank the Taiwan branch of Sanofi-Aventis for providing oxaliplatin and docetaxel for this study.

\section{References}

1. Parkin DM. Epidemiology of cancer global patterns and trends. Toxicol Lett. 1998;102:227-34.

2. Alberts SR, Cervantes A, Van de Velde CJH. Gastric cancer: epidemiology, pathology and treatment. Ann Oncol. 2003; 14(Suppl 2):ii31-6.

3. Wagner AD, Unverzagt S, Grothe W, Kleber G, Grothey A, Haerting J, et al. Chemotherapy for advanced gastric cancer. Cochrane Database Syst Rev. 2010;3:CD004064.

4. Pozzo C, Barone C. Is there an optimal chemotherapy regimen for the treatment of advanced gastric cancer that will provide a platform for the introduction of new biological agents? Oncologist. 2008;13:794-806.

5. Eisenhauer EA, Vermorken JB. The taxoids. Comparative clinical pharmacology and therapeutic potential. Drugs. 1998;55: 5-30.

6. Sulkes A, Smyth J, Sessa C, Dirix LY, Vermorken JB, Kaye S, et al. Docetaxel (Taxotere) in advanced gastric cancer: results of a phase II clinical trial. EORTC Early Clinical Trials Group. Br J Cancer. 1994;70:380-3.

7. Nishiyama M, Wada S. Docetaxel: its role in current and future treatment for advanced gastric cancer. Gastric Cancer. 2009;12: $132-41$.

8. Van Cutsem E, Moiseyenko VM, Tjulandin S, Tjulandin S, Majlis A, Constenla M, et al. Phase III study of docetaxel and cisplatin plus fluorouracil compared with cisplatin and fluorouracil as first-line therapy for advanced gastric cancer: a report of the V325 study group. J Clin Oncol. 2006;24:4991-7.

9. Hainsworth JD, Howard AB III, Erland JB, Thomas M, Greco FA. Phase I trial of docetaxel administered by weekly infusion in 
patients with advanced refractory cancer. J Clin Oncol. 1998; 16:2164-8.

10. Camps C, Massuti B, Jimenez A, Maestu I, Gómez RG, Isla D, et al. Randomized phase III study of 3-weekly versus weekly docetaxel in pretreated advanced non-small-cell lung cancer: a Spanish Lung Cancer Group trial. Ann Oncol. 2006;17:467-72.

11. Graziano F, Catalano V, Baldelli AM, Giordani P, Testa E, Lai V, et al. A phase II study of weekly docetaxel as salvage chemotherapy for advanced gastric cancer. Ann Oncol. 2000;11:1263-6.

12. Kweekel DM, Gelderblom H, Guchelaar HJ. Pharmacology of oxaliplatin and the use of pharmacogenomics to individualize therapy. Cancer Treat Rev. 2005;31:90-105.

13. Cunningham D, Starling N, Rao S, Iveson T, Nicolson M, Coxon F, et al. Capecitabine and oxaliplatin for advanced esophagogastric cancer. N Engl J Med. 2008;358:36-46.

14. Al-Batran SE, Hartmann JT, Probst S, Schmalenberg H, Hollerbach S, Hofheinz R, et al. Phase III trial in metastatic gastroesophageal adenocarcinoma with fluorouracil, leucovorin plus either oxaliplatin or cisplatin: a study of the Arbeitsgemeinschaft Internistische Onkologie. J Clin Oncol. 2008;26:1435-42.

15. Richards D, McCollum D, Wilfong L, Sborov M, Boehm KA, Zhan F, et al. Phase II trial of docetaxel and oxaliplatin in patients with advanced gastric cancer and/or adenocarcinoma of the gastroesophageal junction. Ann Oncol. 2008;19:104-8.

16. Kim JG, Sohn SK, Chae YS, Song HS, Kwon KY, Do YR, et al. Multicenter phase II study of docetaxel plus oxaliplatin combination chemotherapy in patients with advanced gastric cancer: Daegu Gyeongbuk Oncology Group. Br J Cancer. 2008;98: 542-6.

17. Barone C, Basso M, Schinzari G, Pozzo C, Trigila N, D'Argento $\mathrm{E}$, et al. Docetaxel and oxaliplatin combination in second-line treatment of patients with advanced gastric cancer. Gastric Cancer. 2007;10:104-11.

18. Therasse P, Arbuck SG, Eisenhauer EA, Wanders J, Kaplan RS, Rubinstein L, et al. New guidelines to evaluate the response to treatment in solid tumors. J Natl Cancer Inst. 2001;92:205-16.

19. Kim KH, Park YS, Chang MH, Kim HS, Jun HJ, Uhm J, et al. A phase I/II of docetaxel and oxaliplatin in patients with advanced gastric cancer. Cancer Chemother Pharmacol. 2009;64:347-53.
20. Zhong H, Zhang Y, Ma S, Ying JE, Yang Y, Tong D, et al. Docetaxel plus oxaliplatin (DOCOX) as a second-line treatment after failure of fluoropyrimidine and platinum in Chinese patients with advanced gastric cancer. Anticancer Drugs. 2008;19(10): 1013-8.

21. Ajani JA. Optimizing docetaxel chemotherapy in patients with cancer of the gastric and gastroesophageal junction: evolution of the docetaxel, cisplatin, and 5-fluorouracil regimen. Cancer. 2008;113:945-55.

22. Roth AD, Fazio N, Stupp R, Falk S, Bernhard J, Saletti P, et al. Docetaxel, cisplatin, and fluorouracil; docetaxel and cisplatin; and epirubicin, cisplatin, and fluorouracil as systemic treatment for advanced gastric carcinoma: a randomized phase II trial of the Swiss Group for Clinical Cancer Research. J Clin Oncol. 2007; 5:3217-23.

23. Lo SS, Khorana AA, Javle M, Simon S, Kiefer G, Rajasenan K, et al. A phase II study of weekly docetaxel in combination with capecitabine in advanced gastric and gastroesophageal adenocarcinomas. Oncology. 2010;78:125-9.

24. Jatoi A, Murphy BR, Foster NR, Nikcevich DA, Alberts SR, Knost JA, et al. Oxaliplatin and capecitabine in patients with metastatic adenocarcinoma of the esophagus, gastroesophageal junction and gastric cardia: a phase II study from the North Central Cancer Treatment Group. Ann Oncol. 2006;17:29-34.

25. Park YH, Kim BS, Ryoo BY, Yang SH. A phase II study of capecitabine plus 3-weekly oxaliplatin as first-line therapy for patients with advanced gastric cancer. Br J Cancer. 2006;94(7): 959-63.

26. Li CP, Chen JS, Chen LT, Yen CJ, Lee KD, Su WP, et al. A phase II study of weekly docetaxel and cisplatin plus oral tegafur/ uracil and leucovorin as first-line chemotherapy in patients with locally advanced or metastatic gastric cancer. Br J Cancer. 2010; 103(9):1343-8.

27. Bang YJ, Van Cutsem E, Feyereislova A, Chung HC, Shen L, Sawaki A, et al. Trastuzumab in combination with chemotherapy versus chemotherapy alone for treatment of HER2-positive advanced gastric or gastro-oesophageal junction cancer (ToGA): a phase 3, open-label, randomised controlled trial. Lancet. 2010; 376:687-97. 